\author{
Shinsho Oryu (D) Takashi Watanabe • Yasuhisa Hiratsuka • \\ Masayuki Takeda • Yoshio Togawa
}

\title{
An Investigation of the Nuclear Reaction Near the Three-Body Break-up Threshold: As a Ultra Low Energy Nuclear Synthesis
}

Received: 12 March 2019 / Accepted: 30 April 2019 / Published online: 20 May 2019

(C) The Author(s) 2019

\begin{abstract}
We investigate the possibility of lanthanum (La)-nucleus creation via the reaction $\mathrm{Cs}(2 \mathrm{~d}, \gamma) \mathrm{La}$ on the three-ion quasi-molecule $\mathrm{CsD}_{2}$ in the $\mathrm{CsD}_{2} \mathrm{Pd}_{12}$-cluster. In order to calculate $\mathrm{d}-\mathrm{Cs}-\mathrm{d}$ (or D-Cs-D) three-body bound states and wave functions, we adopt a very accurate three-body variational method with more than 80 figures. The $\mathrm{CsD}_{2}$ and La binding energies and wave functions are obtained with an $\mathrm{S}$-wave trial functions. The wave function overlap (WFO) value between the La nuclear excited state and the several $\mathrm{CsD}_{2}$ quasi molecular modified states is calculated. It should be stressed that the WFO value is of critical importance for the existence of the electro-magnetic transition in the $\mathrm{Cs}(2 \mathrm{~d}, \gamma) \mathrm{La}$ reaction. We found that the WFO value is very sensitive to the nuclear potential tail with a two- or three-body $1 / r^{2}$-type long range hadron potential, and gives a promising result for the reaction. The result is also followed by the three-body Faddeev calculation.
\end{abstract}

\section{Introduction}

In the traditional nuclear fusion approach, the most important problem is whether the incident energy can penetrate the Coulomb barrier or not, because the reaction is usually started in the free field. Recently we pointed out that the Cs-d penetration in the quasi-molecule which is developed in the Pd-crystal could easily occur [1], since the energy levels of such a quasi-molecule could close to the top of Cs-d Coulomb barrier or over the barrier in the "Pd-cage" which is a repulsive Coulomb barrier between the ions $(\mathrm{Cs} / \mathrm{D})$ and outer $\mathrm{Pd}$. Therefore, the penetration problem is resolved in the quasi-molecule: $\mathrm{CsD}_{2}$ in the $\mathrm{CsD}_{2} \mathrm{Pd}_{12}$ system, and $\mathrm{CsD}_{4}$ in $\mathrm{CsD}_{4} \mathrm{Pd}_{12}$, and also $\mathrm{CsD}_{6}$ in the system $\mathrm{CsD}_{6} \mathrm{Pd}_{12}$ which are given by the hydrogen storage in the $\mathrm{Pd}_{13}$ cub octahedron/ $\mathrm{D}_{6}$, or Pd-cluster where the central $\mathrm{Pd}$ is substituted by $\mathrm{Cs}$ which was precisely investigated by Watari et al. [2]. In their paper, the numerical calculation was done with the molecular ADF program, which

This article belongs to the Topical Collection "Ludwig Faddeev Memorial Issue".

S. Oryu $(\varangle) \cdot$ T. Watanabe $\cdot$ M. Takeda $\cdot$ Y. Togawa

Tokyo University of Science, 2641 Yamazaki, Noda, Chiba 278-8510, Japan

E-mail: oryu@ @s.noda.tus.ac.jp

T. Watanabe

E-mail: a14004@rs.tus.ac.jp

M. Takeda

E-mail: takeda@rs.noda.tus.ac.jp

Y. Togawa

E-mail: togawa@rs.noda.tus.ac.jp

Y. Hiratsuka

Preparation School of HLF Ltd, Kiryu, Gunma 376-0021, Japan

E-mail: yasuhisa1059@gmail.com 


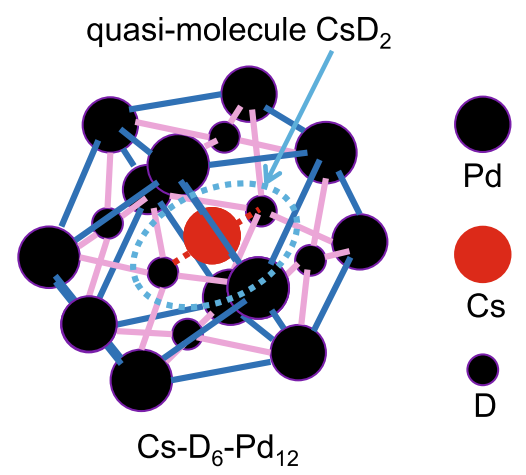

Fig. $1 \mathrm{CsD}_{2}$ quasi-molecule in the $\mathrm{CsD}_{2} \mathrm{Pd}_{12}$ cluster which is based on in the $\mathrm{Pd}_{13}$ cub octahedron/ $\mathrm{D}_{6}$, where one of the central $\mathrm{Pd}$ is substituted by Cs

is based on the linear combination of atomic orbital's method using density functional theory with all-electron Slater-type basis sets [2]. They found that the external electron configuration of $\mathrm{CsD}_{2} \mathrm{Pd}_{12}$ is very similar to that of $\mathrm{La}$ [2]. Therefore one could confirm that such a quasi-molecule exists independently from the Pd-cluster. By this reason, we call $\mathrm{CsD}_{2}$ in the $\mathrm{CsD}_{2} \mathrm{Pd}_{12}$ crystal the quasi molecule (see Fig. 1). However, their calculation was done in the region of $10^{4} \mathrm{fm} \leq r<\infty$, therefore, it is very hard to investigate the nuclear fusion problem which is performed in the region of $0 \leq r \leq \infty$.

On the other hand, in the nuclear cluster physics, it is known that the highest excited state of the nucleus is the linear chain of the clusters [3-9], which is very similar to the molecular system in the present case (see Fig. 1). However, such a nuclear energy level is still far from the molecular state. Therefore, one could imagine that the nuclear states with the long range potential, which have predicted in the hadron three-body system [10-14] could interpolate such a region between the nuclear state and the molecular state.

In order to investigate a very shallow nuclear state, we would like to study nuclear d-Cs-d three-body problem by a very accurate three-body variational (HDV) method with 80 to 100 figures based on a usual variational approach [15]. For the nuclear potentials, the WS potential is adopted with the repulsive Coulomb potentials for $\mathrm{Cs}-\mathrm{D}, \mathrm{D}-\mathrm{D}$, and the repulsive Pd-cage, and also we have to take into account the electron effects for the attractive $\mathrm{Ze}$-Cs, $\mathrm{Ze}$-D Coulomb potentials.

The total Hamiltonian of the quasi-molecule D-Cs-D with the Pd-cage and the nuclear d-Cs-d (or D-Cs-D) system is discussed in Sect. 2. In Sect. 3, the quasi molecular low-lying wave function $\psi_{m}$ and the nuclear wave function of the highest excited state of La $\psi_{n}$ are obtained for the reaction: $\mathrm{Cs}(2 \mathrm{~d}, \gamma) \mathrm{La}$. The WFO value between both wave functions: $\psi_{n}$, and $\psi_{m}$ will be compared for the cases where the two- and three-body long range potentials are included or not. The conclusion and discussion will be given in Sect. 4 .

\section{Effective Three-Body Hamiltonian In Molecular D-Cs-D and Nuclear d-Cs-d Systems in Pd-Cluster}

Since some electrons could be contained between Cs and d nuclei in the molecular system, therefore we use the sign D-Cs-D for the three-ion system, while we take a sign $d-C s-d$ for the nuclear system where no electron exists between Cs and d. Therefore, hereafter we will frequently use " $H$ " instead of "D and d", and also use "N" for nucleus or ions $\{\mathrm{Cs}, \mathrm{D}$ or $\mathrm{d}\}$ without confusion. The two-body Coulomb potentials in the $\mathrm{H}-\mathrm{Cs}-\mathrm{H}$ three-body system are given by the $\mathrm{Cs}-\mathrm{H}$ potential: $V_{c}^{\mathrm{CsH}}$, and the $\mathrm{H}-\mathrm{H}$ potential: $V_{c}^{\mathrm{HH}}$, the sum of the attractive Coulomb potentials between $Z$-electrons $(Z e l)$ and ion: $V_{\mathrm{N}-Z e l}^{M}$ where $\mathrm{N}$ stands for $\{\mathrm{Cs}, \mathrm{D}\}$ ions, and the electron-electron Coulomb repulsive potential: $V_{\mathrm{el}-\mathrm{el}}^{M}$ which is the sum of the $i$ th-electron and the $j$ th-electron: $V_{c}^{\mathrm{e}_{i}-\mathrm{e}_{j}}$. For the three-nuclear (hadron) system: d-Cs-d, the two-body nuclear potentials are given by $V_{3-\mathrm{N}}^{\text {had }}$ especially for the Cs-d potential: $V_{N}^{\mathrm{Csd}}$, and for the d-d potential: $V_{N}^{\mathrm{dd}}$. The two-body Coulomb interactions among three ions: $V_{3-\mathrm{N}}^{C}$, especially for $\mathrm{Cs}-\mathrm{H}$ interaction: $V_{c}^{\mathrm{CsH}}$, and also for $\mathrm{H}-\mathrm{H}$ interaction: $V_{c}^{\mathrm{HH}}$, respectively.

Therefore the total Hamiltonian of $\mathrm{H}-\mathrm{Cs}-\mathrm{H}$ quasi-molecule in the $\mathrm{Pd}$-cluster is given by using the repulsive Coulomb barrier with the one-body potential: $V_{c}^{\mathrm{Pd}}$, and by adding three-cluster force to fit the ground state of 
La: $V_{t}$, and also with a two- or three-body long range hadron interaction: $V_{e}$ if any,

$$
\begin{aligned}
H & =\left(K_{3-\mathrm{N}}+V_{3-\mathrm{N}}^{h a d}+V_{3-\mathrm{N}}^{C}+V_{c}^{\mathrm{Pd}}+V_{t}+V_{e}\right)+\left(K_{\mathrm{Z}-\mathrm{el}}+V_{\mathrm{N}-\mathrm{Zel}}^{M}+V_{\mathrm{el}-\mathrm{el}}^{M}\right) \\
& =H_{\text {had }}+h_{c}^{M},
\end{aligned}
$$

where $K_{3-\mathrm{N}}$ and $K_{\mathrm{Z}-\mathrm{el}}$ are the three-hadron kinetic energy, and $Z$-electron kinetic energy, respectively. The total Hamiltonian consists of the hadron part and the electron part,

$$
\begin{aligned}
H_{\text {had }}= & K_{3-\mathrm{N}}+V_{3-\mathrm{N}}^{\text {had }}+V_{3-\mathrm{N}}^{C}+V_{c}^{\mathrm{Pd}}+V_{t}+V_{e} \\
& h_{c}^{M}=K_{\mathrm{Z}-\mathrm{el}}+V_{\mathrm{N}-\mathrm{Zel}}^{M}+V_{\mathrm{el}-\mathrm{el}}^{M}
\end{aligned}
$$

where the potentials are defined by using coordinates: $\mathbf{r}_{i j}=\mathbf{r}_{i}-\mathbf{r}_{j}$, for three-different ions $\mathbf{r}_{1}, \mathbf{r}_{2}$ and $\mathbf{r}_{3}$,

$$
\begin{gathered}
V_{3-\mathrm{N}}^{\text {had }}\left(\mathbf{r}_{1}, \mathbf{r}_{2}, \mathbf{r}_{3}\right)=\left[V_{N}^{\mathrm{Csd}_{1}}\left(\mathbf{r}_{31}\right)+V_{N}^{\mathrm{Csd}_{2}}\left(\mathbf{r}_{23}\right)+V_{N}^{\mathrm{d}_{1} \mathrm{~d}_{2}}\left(\mathbf{r}_{12}\right)\right] \\
V_{3-\mathrm{N}}^{C}\left(\mathbf{r}_{1}, \mathbf{r}_{2}, \mathbf{r}_{3}\right)=\left[V_{c}^{\mathrm{Csd}_{1}}\left(\mathbf{r}_{31}\right)+V_{c}^{\mathrm{Csd}_{2}}\left(\mathbf{r}_{23}\right)+V_{c}^{\mathrm{d}_{1} \mathrm{~d}_{2}}\left(\mathbf{r}_{12}\right)\right] \\
V_{\mathrm{N}-\mathrm{Zel}}^{M}\left(\mathbf{r}_{1}, \mathbf{r}_{2}, \mathbf{r}_{3}\right)=\left[V_{c}^{\mathrm{ZeD}_{1}}\left(\mathbf{r}_{1}\right)+V_{c}^{\mathrm{ZeD}_{2}}\left(\mathbf{r}_{2}\right)+V_{c}^{\mathrm{ZeCs}}\left(\mathbf{r}_{3}\right)\right] \\
V_{\mathrm{el}-\mathrm{el}}^{M}=\sum_{i=1}^{Z} \sum_{j \neq i} V_{c}^{\mathrm{e}_{i}-\mathrm{e}_{j}} \\
V_{c}^{\mathrm{Pd}}\left(\mathbf{r}_{1}, \mathbf{r}_{2}, \mathbf{r}_{3}\right)=V_{c}^{\mathrm{PdCs}}\left(\mathbf{r}_{3}\right)+V_{c}^{\mathrm{PdD}_{1}}\left(\mathbf{r}_{1}\right)+V_{c}^{\mathrm{PdD}_{2}}\left(\mathbf{r}_{2}\right) .
\end{gathered}
$$

$V_{c}^{\mathrm{Pd}}\left(\mathbf{r}_{1}, \mathbf{r}_{2}, \mathbf{r}_{3}\right), V_{t}\left(\mathbf{r}_{1}, \mathbf{r}_{2}, \mathbf{r}_{3}\right)$, and $V_{e}\left(\mathbf{r}_{1}, \mathbf{r}_{2}, \mathbf{r}_{3}\right)$ are a one-body potential of the Pd Coulomb barrier, the threecluster nuclear potential, and a long range three-body potential if any. Equation (7) is represented only by the operator form which should be given by the different arguments for $(Z+2)$-electron degrees of freedom.

In Eq. (4), we adopt the WS potential for the $i$ th-N and $j$ th-N nuclear potential: $V_{N}^{\mathrm{N}_{i} \mathrm{~N}_{j}}\left(\mathbf{r}_{i j}\right)$,

$$
V_{W}^{\mathrm{N}_{i} \mathrm{~N}_{j}}\left(r_{i j}\right)=V_{W 0}^{\mathrm{N}_{i} \mathrm{~N}_{j}}\left[1+\exp \left(\frac{r_{i j}-R_{W}^{\mathrm{N}_{i} \mathrm{~N}_{j}}}{a_{W}^{\mathrm{N}_{i} \mathrm{~N}_{j}}}\right)\right]^{-1}
$$

with parameters, $V_{W 0}^{\mathrm{Csd}}=-79.30 \mathrm{MeV}, V_{W 0}^{\mathrm{dd}}=-27.57 \mathrm{MeV}, R_{W}^{\mathrm{Csd}}=10.21 \mathrm{fm}, R_{W}^{\mathrm{dd}}=1.49 \mathrm{fm}, a_{W}^{\mathrm{Csd}}=$ $0.4 \mathrm{fm}, a_{W}^{\mathrm{dd}}=0.3 \mathrm{fm}$, respectively.

In Eq. (5), we have

$$
V_{c}^{\mathrm{N}_{i} \mathrm{~N}_{j}}\left(r_{i j}\right)= \begin{cases}\frac{Z_{i} Z_{j} e^{2}}{8 \pi R}\left[3-\left(\frac{r_{i j}}{R_{c}^{\mathrm{N}_{i} \mathrm{~N}_{j}}}\right)^{2}\right] & \text { for } r_{i j} \leq R \\ \frac{Z_{i} Z_{j} e^{2}}{4 \pi r_{i j}} Q & \text { for } R<r_{i j}\end{cases}
$$

with $R_{c}^{\mathrm{CsH}}=10.21 \mathrm{fm}$, and $R_{c}^{\mathrm{H}_{1} \mathrm{H}_{2}}=1.49 \mathrm{fm}$.

In Eq. (7), the electron-electron interaction may be attributed to the electron density function that is responsible for the potential in Eq. (6). Therefore, we can omit Eq. (7) in our three-body calculation. Furthermore, the attractive Coulomb potential between the $Z e$-charged ion and the $-Z^{\prime} e$-charged electron cloud constructs the $\mathrm{CsD}_{2} \mathrm{Pd}_{12}$ cluster with the characteristic lattice constants. Therefore, we can also omit the potential Eq. (6) when we take into account our three-cluster system. Although, the potential is formally given by using the 
electron density function $\rho(r)$ with the fine structure constant $\alpha$,

$$
\begin{aligned}
V_{c}^{Z^{\prime} N_{i}}\left(r_{i}\right)= & Z_{i} \hbar c \alpha\left[\frac{4 \pi}{r_{i}} \int_{0}^{r_{i}} \rho\left(r^{\prime}\right) r^{\prime 2} d r^{\prime}+4 \pi \int_{r_{i}}^{\infty} \rho\left(r^{\prime}\right) r^{\prime} d r^{\prime}\right] \\
= & Z_{i} \hbar c \alpha\left[\frac{4 \pi}{r_{i}} \int_{0}^{\infty} \rho\left(r^{\prime}\right) r^{\prime 2} d r^{\prime}-\frac{4 \pi}{r_{i}} \int_{r_{i}}^{\infty} \rho\left(r^{\prime}\right) r^{\prime 2} d r^{\prime}\right. \\
& \left.+4 \pi \int_{r_{i}}^{\infty} \rho\left(r^{\prime}\right) r^{\prime} d r^{\prime}\right] \\
= & -Z_{i} \hbar c \alpha\left[\frac{Z^{\prime}}{r_{i}}+\frac{4 \pi}{r_{i}} \int_{r_{i}}^{\infty} \rho\left(r^{\prime}\right)\left\{r^{\prime 2}-r^{\prime} r_{i}\right\} d r^{\prime}\right],
\end{aligned}
$$

where the electron distribution is given by,

$$
\rho(r)=\sum_{i=1}^{M} c_{i} \exp \left[-\left(\frac{r}{R_{i}^{e}}\right)^{2}\right],
$$

with

$$
4 \pi \int_{0}^{\infty} \rho\left(r^{\prime}\right) r^{\prime 2} d r^{\prime}=-Z^{\prime},
$$

where $Z^{\prime}$ is variable by the electron supply from $\mathrm{Pd}$-cage.

In Eq. (8), the Pd-N $\mathrm{N}_{i}$ Coulomb barrier is represented by a one-body potential,

$$
V_{c}^{\mathrm{PdN}_{i}}\left(r_{i}\right)=V_{c 0}^{\mathrm{Pd}}\left(\frac{r_{i}}{a_{c}^{\mathrm{Pd}}}\right)^{10} \exp \left\{-\left(\frac{r_{i}-a_{c}^{\mathrm{Pd}}}{b_{c}^{\mathrm{Pd}}}\right)^{2}\right\}
$$

with $V_{c 0}^{\mathrm{Pd}}=1.0 \times 10^{-4} \mathrm{MeV}, a_{c}^{\mathrm{Pd}}=5.0 \times 10^{5} \mathrm{fm}$, and $b_{c}^{\mathrm{Pd}}=3.1623 \times 10^{5} \mathrm{fm}$, respectively. These parameters for the Pd-cage indicate that the location of Pd is $1.57 \times 10^{6} \mathrm{fm}$, and $2.73 \mathrm{MeV}$ height, however, these values may vary.

In this paper, we adopt a three-cluster potential as,

$$
V_{t}\left(r_{1}, r_{2}, r_{3}\right)=V_{t 0} \exp \left[-\left(\frac{r_{1}-r_{2}}{a_{t}}\right)^{2}-\left(\frac{r_{2}-r_{3}}{a_{t}}\right)^{2}-\left(\frac{r_{3}-r_{1}}{a_{t}}\right)^{2}\right],
$$

where $V_{t 0}=1800 \mathrm{MeV}$ and $a_{t}=3.0 \mathrm{fm}$ are used to fit the ground state of La by adding the Coulomb force. We obtain a very good fit to the experimental ground state energy $\mathcal{E}_{0}=-32.3 \mathrm{MeV}$, the root mean square (rms) radius $R_{0}=6.25 \mathrm{fm}$, the highest excited energy $\mathcal{E}_{\max }=-3.5134 \times 10^{2} \mathrm{keV}$ for the negative value, and the rms radius $R_{\max }=165.5 \mathrm{fm}$, for the usual nuclear potential with the Coulomb plus three-cluster force by the HDV calculation. Therefore, the highest energy level is far from that of the usual molecular state, although the higher partial waves and the non-central part of the WS potential have been omitted for the first time.

On the other hand, in order to obtain shallow energy levels that could be close to the molecular states we adopt a kind of three-body long range potential with $1 / r_{i j}^{n}$ tail which was proposed as the GPT potential [10-14] with a Lorentz form or given by the modified Efimov type [16-18]:

$$
V_{e}\left(r_{1}, r_{2}, r_{3}\right)=V_{e 0} a_{e}^{n}\left[\left(r_{1}-r_{2}\right)^{n}+\left(r_{2}-r_{3}\right)^{n}+\left(r_{3}-r_{1}\right)^{n}+a_{e}^{n}\right]^{-1},
$$

where we choose $n=2$ in this paper. Parameters $V_{e 0}=-80,000 \mathrm{MeV}$, and $a_{e}=5000 \mathrm{fm}$ are taken, and also a supplemental $a_{e}^{n}$ is used to avoid divergence. It could be noticed that these parameters seem to be too 


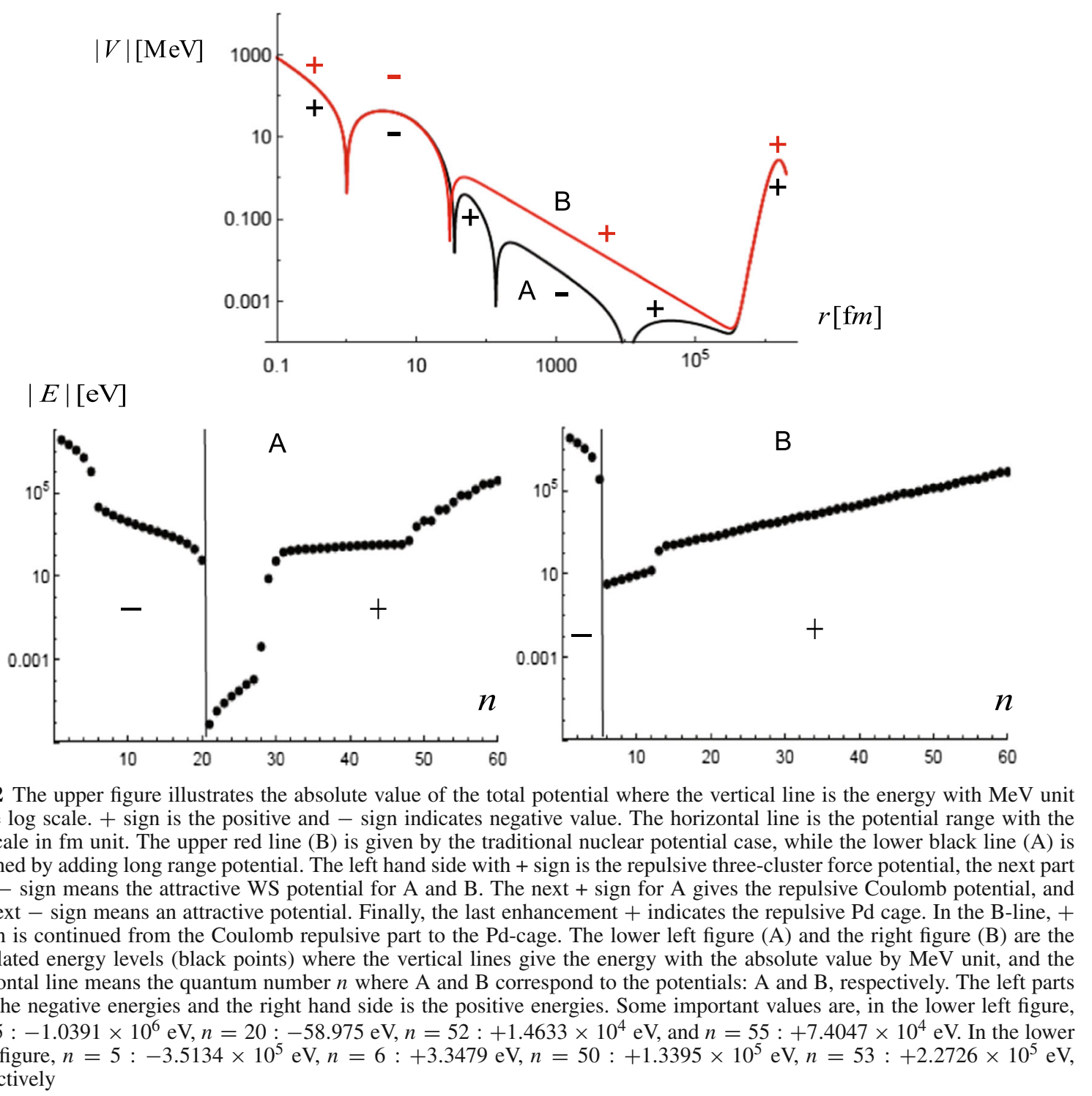

large to compare to the parameters in the WS potential, however Eq. (18) does not change the usual nuclear potential in the shorter range, because $V_{e}$ and $V_{t}$ are complementary.

On the $k$ th-particle transfer diagram in the three-body AGS Born term [19], $V_{e}$ becomes (A) $V_{e}=V_{e 0} a_{e}^{2} /\left[r_{i j}^{2}+\right.$ $\left.a_{e}^{2}\right]$ for $\left(r_{k i}=0\right.$ and $\left.r_{j k}=0\right), V_{e}=V_{e 0} a_{e}^{2} /\left[2 r_{i j}^{2}+a_{e}^{2}\right]$ for $\left(r_{k i}=0\right.$ or $\left.r_{j k}=0\right)$ which are the three-body long range potential between a pair and the spectator particle; and (B) $V_{e}=V_{e 0} a_{e}^{2} /\left[2 r_{k j}^{2}+a_{e}^{2}\right]=V_{e 0} a_{e}^{2} /\left[2 r_{k i}^{2}+a_{e}^{2}\right]$ (for $r_{i j}=0$ ) which indicates the two-body long range potential. (C) While, putting $\mathbf{r}_{k}=\left(\mathbf{r}_{i}+\mathbf{r}_{j}\right) / 2$, it gives $V_{e}=V_{e 0} a_{e}^{2} /\left[3 r_{i j}^{2} / 2+a_{e}^{2}\right]$ which is due to the three-body long range potential as well as (A) [10].

Therefore, the D-Cs-D system is given by a wine bottle-type potential which is represented by Eqs. (9), (10), (16), (17), and (18) (see Fig. 2). Figure 2 represents the half of the wine bottle-type potential. The constituents of the quasi molecule $\mathrm{CsD}_{2}$ align in this potential, where $\mathrm{Cs}$ is located in the center of the potential, which are the highly resonated La nucleus $\mathrm{d}-\mathrm{Cs}-\mathrm{d}$, where the electrons could encircle the $\mathrm{CsD}_{2}$ and rather stick to the Pd-cage. 


\section{Numerical Results}

The two-cluster potential between Cs and d could be constructed by the three-body reaction: $\mathrm{Cs}+\mathrm{d} \equiv$ $\mathrm{Cs}+(\mathrm{N}, \mathrm{N}) \rightleftharpoons(\mathrm{Cs}, \mathrm{N})+\mathrm{N}$ which gives the general particle transfer (GPT) potential [14] or the AGS Born term [19]. However, in such a way, it is very hard to make the two-cluster form-factors with the core excitation for the three-cluster problem: d-Cs-d. Therefore, we adopt the WS-type Cs-d potential of Eq. (9). By using the WS and the Coulomb potentials, the two-body energy levels are shown in Table 2, where the ground states are well fitted. It is found that the number of the calculated energy levels considering only the central part of the WS potential are rather small. Therefore, additional WS components should be incorporated containing for example spin-orbit and tensor terms for the two-cluster interactions.

Especially, we know the four-nucleon Faddeev-Yakubovsky equations for the deuteron-deuteron interaction [20]. However, our aim in this paper is to obtain the highest three-cluster (or -ion) nuclear level, where the shallow energy levels should be well introduced by the S-wave or even central potential. Furthermore, we obtained the La ground state $-32.3 \mathrm{MeV}$ (Table 3 ) for $\mathrm{d}+\mathrm{Cs}+\mathrm{d}$ system by using the central two-cluster $\mathrm{Cs}-\mathrm{d}$ and $\mathrm{d}-\mathrm{d}$ potentials, and with the three-cluster force Eq. (17), where we confirmed that the phenomenological method for the La ground state is not very sensitive to the shallow bound and/or resonance states.

The traditional nuclear interaction appears in the region of $0 \leq r<10^{4} \mathrm{fm}$, however, the molecular levels are calculated in the region of $10^{4} \mathrm{fm} \leq r<10^{7} \mathrm{fm}$. Therefore, the nuclear wave functions and the molecular wave functions do not overlap to give rise to the electro-magnetic (EM) transition. This means that the molecular states are stable and never go down to the nuclear states.

However, the binding energy of $\mathrm{CsD}_{2}$ in the $\mathrm{Pd}_{12}$-cage is completely different from the free field. We solve the three-ion system in the $\operatorname{Pd}_{12}$-cage for the full region: $0 \leq r<\infty$ by the HDV method. In other words, for the nuclear and the molecular systems we can solve the eigen-equation on the basis of a common field in the full region with the 80-100 figures accuracy, however it seems to be very hard for the usual FORTRAN program in this stage. The second promising method is shown in Ref. [1] where the entire wave function could be made by the nuclear state and the molecular state: $|\Psi\rangle=\left|\psi \otimes \psi_{M}\right\rangle$. Both energy states could be corrected by the boost up effect which is shown in Ref. [1], however we do not take this method in this paper.

We compared the calculated results for two potentials,

Table 1 Some related ions and the shallow spin-parities: $J^{\pi}$, and masses by $(\mathrm{u})$ and $(\mathrm{MeV})$ units, where $1 \mathrm{u}=931.5019 \mathrm{MeV}$ is the universal constant

\begin{tabular}{llll}
\hline Ions & $J^{\pi}$ & Mass (u) & Mass $($ MeV) \\
\hline${ }^{2} \mathrm{H}$ (deuteron) & $1^{+}$ & 2.0141 & 1876.1 \\
${ }^{4} \mathrm{He}$ & $0^{+}$ & 4.0026 & 3728.4 \\
${ }^{135} \mathrm{Cs}$ & $7 / 2^{+}$ & 134.91 & $1.2567 \times 10^{5}$ \\
${ }^{137} \mathrm{Ba}$ & $3 / 2^{+}$ & 136.91 & $1.2753 \times 10^{5}$ \\
${ }^{139} \mathrm{La}$ & $7 / 2^{+}$ & 138.91 & $1.2939 \times 10^{5}$ \\
\hline
\end{tabular}

Table 2 Calculated two-body binding energies by using the WS potential with the above mentioned parameters and the Coulomb potential, where $E_{1}$ values are compared with the 2-body type in Table 3

\begin{tabular}{llllr}
\hline reaction type & $E_{1}(\mathrm{MeV})$ & $E_{2}(\mathrm{MeV})$ & $E_{3}(\mathrm{MeV})$ & $E_{4}(\mathrm{MeV})$ \\
\hline $\mathrm{d}-\mathrm{d}$ & -23.772716 & & & \\
$\mathrm{Cs}-\mathrm{d}$ & -16.31894 & -13.43531 & -9.003575 & -2.975595 \\
\hline
\end{tabular}

$E_{2}, E_{3}, E_{4}$ are excited states. It should be reminded that these results are obtained only by the central force of the WS potential

Table 3 Some reaction types of binding energies by the mass relation using Table 1 with (MeV) unit

\begin{tabular}{llr}
\hline Reaction type & \multicolumn{1}{c}{ Binding energy } & $(\mathrm{MeV})$ \\
\hline 2-Body & $2 \times m_{{ }^{2} \mathrm{H}}-m_{4} \mathrm{He}$ & 23.8 \\
2-Body & $m_{2} \mathrm{H}+m_{135} \mathrm{Cs}-m_{137} \mathrm{Ba}$ & 16.1 \\
3-Body & $2 \times m_{2} \mathrm{H}+m_{135} \mathrm{Cs}-m_{139} \mathrm{La}$ & 32.3 \\
3-Body & $m_{4} \mathrm{He}+m_{135} \mathrm{Cs}-m_{139} \mathrm{La}$ & 8.4 \\
\hline
\end{tabular}


(1) The first one is the three-body calculation for the full potential with the traditional short range WS potential,

$$
V_{3-\mathrm{N}}^{h a d}+V_{3-\mathrm{N}}^{C}+V_{c}^{\mathrm{Pd}}+V_{t} .
$$

(2) The second one is the three-body calculation for the full potential with the two- and three-body long range potential $V_{e}$ :

$$
V_{3-\mathrm{N}}^{h a d}+V_{3-\mathrm{N}}^{C}+V_{c}^{\mathrm{Pd}}+V_{t}+V_{e} .
$$

The calculated results by the HDV method show a series of negative binding energy levels which starts from $-32.3 \mathrm{MeV}$ to the negative highest nuclear level: $\mathcal{E}_{\max }=-3.5134 \times 10^{5} \mathrm{eV}$ for the short range Eq. (19), and to $\mathcal{E}_{\max }=-58.975 \mathrm{eV}$ for the long range Eq. (20). On the other hand, there is a series of the positive energy states over the Coulomb barrier through to the Pd-cage which is located at $1.57 \times 10^{6} \mathrm{fm}$ and 2.73 $\mathrm{MeV}$ height. Let us call such positive energy states the "quasi molecular states" conventionally.

Therefore, for Eq. (19) the energy gap between the negative nuclear state and the quasi molecular state is very large. However, the calculations with the long range nuclear potential Eq. (20) give a series of discrete negative energy band from $-32.3 \mathrm{MeV}$ to $-58.975 \mathrm{eV}$ (HDV-method: see Fig. 2), or $-44 \mathrm{eV}$ (Faddeev-method: see Fig. 3, where $-2.9 \mathrm{eV}$ is unstable). Therefore, we can conclude that the three-body Faddeev calculation with the $r$-space method gives qualitatively similar results with those by the HDV method in the negative energy

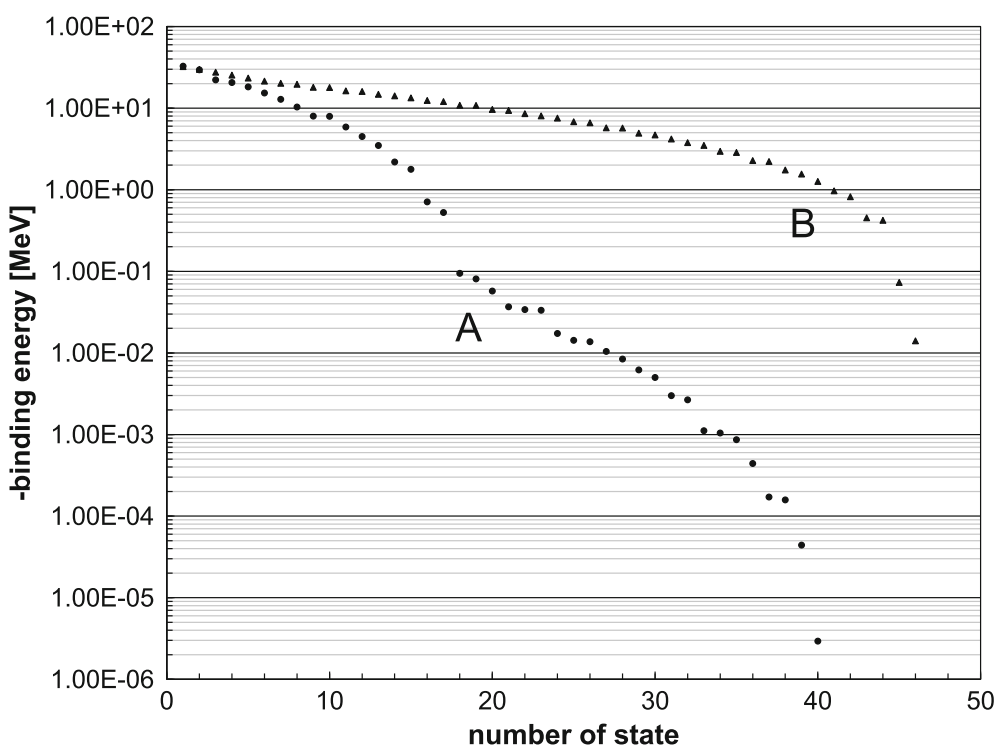

Fig. 3 Faddeev calculations [21] for the negative energy levels: (A) indicates the calculation by Eq. (20), and (B) is given by Eq. (19). The most excited state in (A) is $-2.9 \mathrm{eV}$ which is very close to the molecular levels, however it is not very stable. Therefore, we take the next state: $-44 \mathrm{eV}$ which is relatively stable with two digits accuracy. In the traditional nuclear potential, since the most shallow state $-14 \mathrm{keV}$ in (B) is not stable, then we take a stable $-450 \mathrm{keV}$ which is comparable to HDV result $-351 \mathrm{keV}$, and far from the molecular state

Table 4 Negative highest excited state of La binding energy $\left(\mathcal{E}_{\text {max }}\right)$, and the positive energy $\left(\mathcal{E}_{+}\right)$which give max- $W_{n m}$, and the value of $\max -W_{n m}$ (maximum value of WFO)

\begin{tabular}{llllc}
\hline potentials & $\mathcal{E}_{\max }(\mathrm{eV})$ & $\mathcal{E}_{+}$for max- $W_{n m}(\mathrm{eV})$ & $\max -W_{n m}$ & $W_{n}$ \\
\hline Equation (20) (L) & $-58.975(n=20)$ & $1.4633 \times 10^{4}(m=52)$ & 0.10 & 0.070 \\
Equation (19) (S) & $-3.5134 \times 10^{5}(n=5)$ & $2.2726 \times 10^{5}(m=53)$ & 0.03 & 0.0057 \\
(L)/(S) & $1.6786 \times 10^{-4}$ & $6.4389 \times 10^{-2}$ & 3.33 & 12.3 \\
\hline
\end{tabular}

WFO is defined by: $W_{n m}=\sum_{k=1}^{3}<\psi_{n}\left|o_{k}\right| \psi_{m}>\rightarrow \sum_{k=1}^{3}<\psi_{n}\left|Q_{k} r_{k}^{2}\right| \psi_{m}>$, where $o_{k}$, and $Q_{k}$ stand for a part of the E2 transition operator and the charge of the $k$-th cluster: $o_{k}=Q_{k}\left(2 z_{k}^{2}-x_{k}^{2}-y_{k}^{2}\right)=Q_{k} r_{k}^{2}\left(2 \cos ^{2} \theta_{k}-\sin ^{2} \theta_{k}\right)$ which is replaced by the order of $Q_{k} r_{k}^{2}$. Total WFO: $W_{n} \equiv \sum_{m=n+1}^{60} W_{n m}$ is shown. Calculated results by Eq. (20) is compared with that of Eq. (19), together with the quantum number $n, m$ 
region, although the Faddeev method [21] is rather hard to reach the Pd-cage. These results show that the energy gap between the negative nuclear state and the quasi molecular state is rather small for the long range nuclear potential.

Finally, in Table 4, the WFO results of max- $W_{n m}$ with the long range potential are 3.33 times, and of $W_{n}$ with 12.3 times larger values than those with the usual short range nuclear potential. Therefore, the long range nuclear potential could be favorable to bring about the low energy $\mathrm{Cs}(2 \mathrm{~d}, \gamma)$ La reaction.

\section{Conclusion and Discussion}

This work is based on the assumption that a quasi-molecular system exists in a Pd-cluster. Such a quasimolecule is almost isolated in the Pd-cage. Therefore, the excited state of the quasi-molecule: $\mathrm{CsD}_{2}$ does not break-up, because it is not in the free space but restricted in the Pd-cage. Therefore, some excited states could easily penetrate the Coulomb barrier of the Cs-D interaction or even over the top of it. Therefore, they could go into the nuclear quasi-resonance states by means of "stable" states in the Pd-cage. Hence, a quasi-resonance wave packet of $\mathrm{CsD}_{2}$ could overlap with that of the highest nuclear excited state or the most shallow bound state of La nucleus. In such a case, the WFO has a non zero value, allowing the EM transitions to occur. The possible transition will be started from the E2 or M1 transition, because the E1 transition is forbidden by the same $J^{\pi}$ values for Cs and La. Once, the transfer to the nuclear state occurs, then the nuclear transition to the next states is automatically continued. In our present calculations for the ordinary short range nuclear potential, and for the nuclear potential with a long range, we found that the former case gives a small WFO, but the latter case could offer a larger value by an order of magnitude. We mentioned that the WFO value is critical for the occurrence of the $\operatorname{Cs}(2 \mathrm{~d}, \gamma)$ La reaction.

Recently, one of the authors (S.O) proposed a new long range nuclear potential of the form: $1 / r^{n}$ [10-14]. We pointed out why such a long range part in the one pion transfer interaction or the Yukawa potential comes from [14]. Our conclusion was that any particle transfer in the three-body Faddeev theory [19] generates not only the short range, but also the long range interactions which was called the general particle transfer (GPT) potential. Therefore, any two-body nuclear potential in the three-body constituents is constructed by the GPT potential [10-14]. Furthermore, the three-body problem with the GPT potential generates also a GPT potential between the two-body center of mass and the spectator. This idea is concretely represented by the potential form of Eq. (18). If the long range part contains a $1 / r^{2}$-type potential, the scattering length will become infinity [16-18]. The GPT-potential with the $1 / r^{2}$-type gives the three-body bound states near at the three-body threshold which would be very close to the molecular states.

There are some reports of the experimental results about the low temperature nuclear transition (LTNT) [2228]. Unfortunately, it seems that a reasonable theoretical description for such reactions has not been given as of yet, since almost all of the traditional theories are based on the short range nuclear forces which could reach as far as $100 \mathrm{fm}$. Therefore, the nuclear reaction rate is too small for the LTNT, where the Coulomb repulsive force dominates the attractive nuclear force. Our theory may give a hint to the experimental physicists to investigate a mysterious phenomena such as inexplicable thermal generation measured by a special arrangement in the materials, without any usual nuclear reaction phenomena or radioactive substances. One could imagine that such a high energy $\gamma$-ray emission does not come from the slow reaction caused by the condensed states, or the $\gamma$-ray radiation would be diminished due to the multiple Compton scattering off the electrons in the Pd-cluster, although many nuclear physicists still wonder why some radioactive particles or high energy $\gamma$-rays are not observed in the LTNT.

Finally, it should be noticed that the present calculation adopted only the S-wave trial function, and the central nuclear potential. Therefore, we can not give the final conclusion in this paper. However, our results seem to suggest that if the LTNT experiment could be realized, then the long range potential in the nuclear system could be observed.

\footnotetext{
Acknowledgements The authors would like to acknowledge to Drs. N. Watari, N. Hamada, and A. Kodama for valuable discussions regarding the theoretical aspects of the molecular system, as well as I. Toyoda, S. Tsuruga and H. Kakigami for sharing with us the experimental insight. One of the authors (SO) would like to express his thanks to Profs. I. Lagaris and K. Kato for their helpful suggestions. We are indebted to Mitsubishi Heavy Industry (MHI) Co. Ltd. for significant financial support.

Open Access This article is distributed under the terms of the Creative Commons Attribution 4.0 International License (http:// creativecommons.org/licenses/by/4.0/), which permits unrestricted use, distribution, and reproduction in any medium, provided you give appropriate credit to the original author(s) and the source, provide a link to the Creative Commons license, and indicate if changes were made.
} 


\section{References}

1. S. Oryu, T. Watanabe, Y. Hiratsuka, M. Takeda, Y. Togawa, in Proceedings of FB22 Caen France (2018), Springer (2019)

2. N. Watari, S. Ohnishi, Y. Ishii, Hydrogen storage in Pd-clusters. J. Phys. Condens. Matter. 12, 6799-6823 (2000)

3. K. Ikeda, N. Takigawa, H. Horiuchi, The Ikeda diagram. Prog. Theo. Phys. Suppl. Extra Number 464 (1968)

4. H. Kamada, S. Oryu, The three- $\alpha$ Faddeev calculation on ${ }^{12} \mathrm{C}$ bound states with a Pauli correct $\alpha-\alpha$ potential. Prog. Theor. Phys. 76, 1260-1271 (1986)

5. H. Kamada, S. Oryu, 3- $\alpha$ Cluster Faddeev calculation and effects of 3-body force. Nucl. Phys. A463, 347c-352c (1987)

6. S. Oryu, T. Nishino, H. Kamada, Faddeev-Yakubovsky calculation of 4-alpha particle system with realistic alpha-alpha interactions. Acta Phys. Aust. Few Body Syst. Suppl. 1, 198-205 (1987)

7. S. Oryu, H. Kamada, Three- $\alpha$ model calculation of the ${ }^{12} \mathrm{C}$ nucleus by the Faddeev equation and effects of the three-body force. Nucl. Phys. A 493, 91-111 (1989)

8. S. Oryu, H. Kamada, H. Sekine, T. Nishino, H. Sekiguchi, Four- $\alpha$ model calculation for the ${ }^{16}$ O nucleus by the four-body integral equation. Nucl. Phys. A 534, 221-247 (1991)

9. S. Oryu, H. Kamada, H. Sekine, T. Nishino, H. Sekiguchi, Four alpha model calculation for the ${ }^{16} \mathrm{O}$ nucleus by the four-body integral equation. Nucl. Phys. A 534, 221-247 (1991)

10. S. Oryu, Universal structure of the three-body system. Phys. Rev. C 86, 044001 (2012)

11. S. Oryu, Long-range property in time-dependent interaction with three-body structure and new aspect. Annual Report of Quantum Bio-Informatics Center of Tokyo University of Science, vol. 5, pp. 26-34 (2010). "Hi-Tech Research Center" National Project for Private Universities supported by MEXT in 2006

12. S. Oryu, Long range potential component in the NN force. Few-Body Syst. 54, 283-286 (2013)

13. S. Oryu et al., A New Horizon of Few-Body Problems: exact Coulomb treatment and the energy-momentum translation of the three-body Faddeev equation. J. Phys. Conf. Ser. 915, 012001 (2017)

14. S. Oryu, A new feature of the Efimov-like structure in the Hadron system-long range force as a recoil effect. Few-Body Syst. 59, 51 (2018). https://doi.org/10.1007/s00601-018-1373-z

15. M. Kamimura, Nonadiabatic coupled-rearrangement-channel approach to muonic molecules. Phys. Rev. A 38, 621-624 (1988)

16. A.F. Nicholson, Bound states and scattering in an $1 / r^{-2}$ potential. Aust. J. Phys. 15, 174-179 (1962)

17. V. Efimov, Energy levels arising from resonant two-body forces in a three-body system. Phys. Lett. B 33, 563 (1970)

18. V. Efimov, Energy levels of three resonantly interacting particles. Nucl. Phys. A 210, 157 (1973)

19. E.O. Alt, P. Grassberger, W. Sandhas, Reduction of the three-particle collision problem to multi-channel two-particle Lippmann-Schwinger equations. Nucl. Phys. 2, 167-180 (1967)

20. F. Ciesielski, J. Carbonell, Solutions of the Faddeev-Yakubovsky equations for the four nucleons scattering states. Phys. Rev. C 58, 58-74 (1998)

21. I.J. Thompson, F.M. Nunes, B.V. Danilin, FaCE: a tool for three body Faddeev calculations with core excitation. Comput. Phys. Commun. 161, 87-107 (2004)

22. Yasuhiro Iwamura, Mitsuru Sakano, Takehiko Itoh, Elemental analysis of Pd complexes: effects of D2 gas permeation. Jpn. J. Appl. Phys. 41, 4642 (2002)

23. Y. Iwamura, T. Itoh, S. Tsuruga, Increase of reaction products in deuterium permeation-induced transmutation. J. Condens. Matter. Nucl. Sci. 13, 242-252 (2014)

24. Y. Iwamura, T. Itoh, S. Tsuruga, Increase of reaction products in deuterium permeation-induced transmutation. J. Condens. Matter. Nucl. Sci. 13, 242-252 (2014)

25. T. Higashiyama, H. Miyamaru, A. Takahashi, M. Sakano. Replication of MHI transmutation experiment by $\mathrm{D}_{2}$ gas permeation through Pd complex, in Condensed Matter Nuclear Science (World Scientific, 2005), pp. 447-454

26. H. Yamada, S. Narita, S. Taniguchi, T. Ushirozawa, S. Kurihara, M. Higashizawa, H. Sawada, M. Itagaki, T. Odashima, Producing transmutation element on multi-layered pd sample by deuterium permeation, in Condensed Matter Nuclear Science (World Scientific, 2006), pp. 196-205

27. T. Hioki et al., Inductively coupled plasma mass spectrometry study on the increase in the amount of Pr atoms for Cs-ionimplanted $\mathrm{Pd} / \mathrm{CaO}$ multilayer complex with deuterium permeation. Jpn. J. Appl. Phys. 52, 107301 (2013)

28. S. Tsuruga et al., Transmutation tractions induced by deuterium permeation through nano-structured multilayer thin film. Mitsubishi Heavy Ind. Tech. Rep. 52(4), 104-108 (2015)

Publisher's Note Springer Nature remains neutral with regard to jurisdictional claims in published maps and institutional affiliations. 